\title{
Ward identities and high energy scattering amplitudes in string theory
}

\author{
Chuan-Tsung Chan ${ }^{a}$, Pei-Ming Ho ${ }^{b}$, Jen-Chi Lee ${ }^{c}$ \\ ${ }^{a}$ Physics Division, National Center for Theoretical Sciences, Hsinchu, Taiwan, ROC \\ ${ }^{\mathrm{b}}$ Department of Physics, National Taiwan University and National Center for Theoretical Sciences, \\ Taipei, Taiwan, ROC \\ ${ }^{c}$ Department of Electrophysics, National Chiao-Tung University, Hsinchu, Taiwan, ROC
}

Received 8 November 2004; accepted 16 November 2004

Available online 2 December 2004

\begin{abstract}
High-energy limit $\alpha^{\prime} \rightarrow \infty$ of stringy Ward identities derived from the decoupling of two types of zero-norm states in the old covariant first quantized (OCFQ) spectrum of open bosonic string are used to check the consistency of saddle point calculations of high energy scattering amplitudes of Gross and Mende and Gross and Manes. Some inconsistencies of their saddle point calculations are found even for the string-tree scattering amplitudes of the excited string states. We discuss and calculate the missing terms of the calculation by those authors to recover the stringy Ward identities. In addition, based on the tree-level stringy Ward identities, we give the proof of a general formula, which was proposed previously, of all high energy four-point string-tree amplitudes of arbitrary particles in the string spectrum. In this formula all such scattering amplitudes are expressed in terms of those of tachyons as conjectured by Gross. The formula is extremely simple which manifestly demonstrates the universal high energy behavior of the interactions among all string states.
\end{abstract}

(c) 2004 Elsevier B.V. All rights reserved.

PACS: $11.25 .-\mathrm{W}$

E-mail addresses: ctchan@phys.cts.nthu.edu.tw (C.-T. Chan), pmho@ntu.edu.tw (P.-M. Ho), jcclee@cc.nctu.edu.tw (J.-C. Lee). 


\section{Introduction}

The study of high energy behavior of field theories, in particular Yang-Mills theories, was very successful in the early 1970s. In the quantum chromodynamics, for example, the discovery of asymptotic freedom [1] turned out to be one of the most important properties of Yang-Mills theories. On the other hand, the hidden spontaneously broken symmetry becomes evident at high energies. It is thus very tempting to generalize this study to string theory, which certainly contains a huge hidden symmetry. In 1988, Gross and Mende [2] proposed a saddle point method to calculate high energy $\alpha^{\prime} \rightarrow \infty$ fixed angle string scattering amplitudes. They identified a saddle point to the leading order in energy in the calculation of first quantized string scattering amplitudes for all loops in string perturbation theory. Soon after, based on this remarkable calculation, Gross [3] made important conjectures on high energy stringy symmetries. There are two main conjectures of Gross' pioneer work on this subject. The first one is the existence of an infinite number of linear relations among the scattering amplitudes of different string states that are valid order by order in perturbation theory at high energies. The second is that this symmetry is so powerful as to determine the scattering amplitudes of all the infinite number of string states in terms of, say, the dilaton (tachyon for the case of open string) scattering amplitudes. However, the symmetry charges of his proposed stringy symmetries were not understood and the proportionality constants between scattering amplitudes of different string states were not calculated. As we will see soon, all these problems can be solved by using another independent calculation based on the following key idea: the identification of symmetry charges from an infinite number of stringy zero-norm states with arbitrarily high spins in the $O C F Q$ spectrum.

The importance of zero-norm states and their implication on stringy symmetries were first pointed out in the context of the massive $\sigma$-model approach of string theory [4]. Zero-norm states were shown to imply inter-particle symmetries in the first order weak background field approximation which is valid to all energies. On the other hand, zeronorm states were also shown [5] to carry the spacetime $\omega_{\infty}$ symmetry charges of the 2D string theory. Some implications of stringy Ward identities, derived from the decoupling of two types of zero-norm states, on stringy scattering amplitudes were also discussed in [6]. Recently it was discovered that $[7,8]$ the high energy limit of these stringy Ward identities imply an infinite number of linear relations among scattering amplitudes of different string states with the same momenta. These linear relations can be used to fix the proportionality constants algebraically between scattering amplitudes of different string states at each fixed mass level. These proportionality constants were found to be independent of the scattering angle $\phi_{\mathrm{CM}}$ and the loop order $\chi$ of string perturbation theory as was conjectured by Gross [3]. Thus there is only one independent component of high energy stringy scattering amplitudes for each fixed mass level. For the case of string-tree amplitudes, a general formula can even be given to determine all high energy stringy scattering amplitudes for arbitrary mass levels in terms of those of tachyons [7] — another conjecture by Gross [3].

Since the results we obtained from zero-norm states approach $[7,8]$ are more complete than those obtained via the saddle point method in the past $[2,3,11]$, it would be of interest to directly compare these two independent calculations. After a brief review of zero-norm state calculation in Section 2, in Section 3 we will use high energy limits of our stringy 
Ward identities, which are valid to all energies and all loops, as a consistent check of saddle point calculations. Since there is so far no independent rigorous check of saddle point calculation, partially due to the highly nontrivial structure of moduli spaces of general Riemann surfaces, our simple stringy Ward identities serve as the best theoretical test of this saddle point approximation. Surprisingly, some inconsistencies are found for the results of saddle point calculations even for the string-tree scattering amplitudes of the excited string states. We discuss and calculate the missing terms of the calculation by those authors to recover the stringy Ward identities. In Section 4 of this paper, based on the stringy Ward identities, we derive a general formula of all high energy four-point string-tree amplitudes of arbitrary string states. This formula was first proposed in [7]. Here we will give a general proof and present some examples. This formula determines the scattering amplitudes of all the infinite number of string states in terms of tachyon scattering amplitudes. The formula is extremely simple which manifestly demonstrates the universal high energy behavior of the interactions of an infinite number of string states.

\section{Zero-norm state calculation}

Let us begin with a brief review of zero-norm state calculation $[7,8]$. In the OCFQ spectrum of open bosonic string theory, the solutions of physical state conditions include positive-norm propagating states and two types of zero-norm states. The latter are [9]

Type I: $\quad L_{-1}|x\rangle, \quad$ where $L_{1}|x\rangle=L_{2}|x\rangle=0, L_{0}|x\rangle=0$,

Type II: $\quad\left(L_{-2}+\frac{3}{2} L_{-1}^{2}\right)|\tilde{x}\rangle, \quad$ where $L_{1}|\tilde{x}\rangle=L_{2}|\tilde{x}\rangle=0,\left(L_{0}+1\right)|\tilde{x}\rangle=0$.

While type I states have zero-norm at any spacetime dimension, type II states have zeronorm only at $D=26$. In the first quantized approach of string theory, the stringy on-shell Ward identities are proposed to be [6]

$$
\begin{aligned}
\mathcal{T}_{\chi}\left(k_{i}\right)= & g^{2-\chi} \int \frac{D g_{\alpha \beta}}{\mathcal{N}} D X^{\mu} \exp \left(-\frac{\alpha^{\prime}}{2 \pi} \int d^{2} \xi \sqrt{g} g^{\alpha \beta} \partial_{\alpha} X^{\mu} \partial_{\beta} X_{\mu}\right) \\
& \times \prod_{i=1}^{4} v_{i}\left(k_{i}\right)=0,
\end{aligned}
$$

where at least one of the 4 vertex operators corresponds to the zero-norm state. In Eq. (3) $g$ is the string coupling constant, $\mathcal{N}$ is the volume of the group of diffeomorphisms and Weyl rescalings of the worldsheet metric, and $v_{i}\left(k_{i}\right)$ are the on-shell vertex operators with momenta $k_{i}$. The integral is over orientable open surfaces of Euler number $\chi$ parametrized by moduli $\vec{m}$ with punctures at $\xi_{i}$. In this section, we will use stringy Ward identities of the second mass level $m^{2}=4$ as an example to illustrate our approach. The four stringy Ward identities at this mass level were calculated to be [6]

$$
\begin{aligned}
& k_{\mu} \theta_{\nu \lambda} \mathcal{T}_{\chi}^{(\mu \nu \lambda)}+2 \theta_{\mu \nu} \mathcal{T}_{\chi}^{(\mu \nu)}=0, \\
& \left(\frac{5}{2} k_{\mu} k_{\nu} \theta_{\lambda}^{\prime}+\eta_{\mu \nu} \theta_{\lambda}^{\prime}\right) \mathcal{T}_{\chi}^{(\mu \nu \lambda)}+9 k_{\mu} \theta_{\nu}^{\prime} \mathcal{T}_{\chi}^{(\mu \nu)}+6 \theta_{\mu}^{\prime} \mathcal{T}_{\chi}^{\mu}=0,
\end{aligned}
$$




$$
\begin{aligned}
& \left(\frac{1}{2} k_{\mu} k_{\nu} \theta_{\lambda}+2 \eta_{\mu \nu} \theta_{\lambda}\right) \mathcal{T}_{\chi}^{(\mu \nu \lambda)}+9 k_{\mu} \theta_{\nu} \mathcal{T}_{\chi}^{[\mu \nu]}-6 \theta_{\mu} \mathcal{T}_{\chi}^{\mu}=0, \\
& \left(\frac{17}{4} k_{\mu} k_{\nu} k_{\lambda}+\frac{9}{2} \eta_{\mu \nu} k_{\lambda}\right) \mathcal{T}_{\chi}^{(\mu \nu \lambda)}+\left(9 \eta_{\mu \nu}+21 k_{\mu} k_{\nu}\right) \mathcal{T}_{\chi}^{(\mu \nu)}+25 k_{\mu} \mathcal{T}_{\chi}^{\mu}=0,
\end{aligned}
$$

where $\theta_{\mu \nu}$ is transverse and traceless, and $\theta_{\lambda}^{\prime}$ and $\theta_{\lambda}$ are transverse vectors. These are polarizations of zero-norm states. In each equation, we have chosen, say, $v_{2}\left(k_{2}\right)$ to be the vertex operators constructed from zero-norm states at the mass level $m^{2}=4$ and $k_{\mu} \equiv k_{2 \mu}$. In Eqs. (4)-(7), $v_{1}, v_{3}$ and $v_{4}$ can be any string states (including zero-norm states) at any mass level and we have omitted their tensor indices. $\mathcal{T}_{\chi}^{\prime}$ in Eqs. (4)-(7) are $\chi$ th order stringloop amplitudes. It is important to note that Eqs. (4)-(7) are valid to all loop orders $\chi$ and all energies $E$, and are automatically of the identical form in string perturbation theory.

We will use labels 1 and 2 for the incoming particles and 3 and 4 for the outgoing particles. The center of mass scattering angle $\phi_{\mathrm{CM}}$ is defined to be the angle between $\vec{k}_{1}$ and $\vec{k}_{3}$. The leading order Ward identities of Eqs. (4)-(7) in the energy $E$ expansions were calculated to be (we drop loop order $\chi$ here to simplify the notation) $[7,8]$

$$
\begin{aligned}
& \mathcal{T}_{L L T}^{5 \rightarrow 3}+\mathcal{T}_{(L T)}^{3}=0, \\
& 10 \mathcal{T}_{L L T}^{5 \rightarrow 3}+\mathcal{T}_{T T T}^{3}+18 \mathcal{T}_{(L T)}^{3}=0, \\
& \mathcal{T}_{L L T}^{5 \rightarrow 3}+\mathcal{T}_{T T T}^{3}+9 \mathcal{T}_{[L T]}^{3}=0,
\end{aligned}
$$

where the subscripts and superscripts denote the polarizations and energy orders respectively, which will be explained below. A simple calculation shows that

$$
\mathcal{T}_{T T T}^{3}: \mathcal{T}_{L L T}^{3}: \mathcal{T}_{(L T)}^{3}: \mathcal{T}_{[L T]}^{3}=8: 1:(-1):(-1) .
$$

In the above equations, we have defined the normalized polarization vectors of the second string state to be

$$
\begin{aligned}
e_{P} & =\frac{1}{m_{2}}\left(E_{2}, \mathrm{k}_{2}, 0\right)=\frac{k_{2}}{m_{2}}, \\
e_{L} & =\frac{1}{m_{2}}\left(\mathrm{k}_{2}, E_{2}, 0\right), \\
e_{T} & =(0,0,1),
\end{aligned}
$$

in the $\mathrm{CM}$ frame contained in the plane of scattering. $\mathcal{T}_{T T T}=e_{T}^{\mu} e_{T}^{\nu} e_{T}^{\lambda} \mathcal{T}_{\mu \nu \lambda}$, etc. In Eqs. (8)-(10), we have assigned a relative energy power to each amplitude. For each longitudinal $L$ component, the order is $E^{2}$ (the naive order of $e_{L} \cdot k$ is $E^{2}$ ) and for each transverse $T$ component, the order is $E$ (the naive order of $e_{T} \cdot k$ is $E$ ). This is due to the definitions of $e_{L}$ and $e_{T}$ in Eqs. (13) and (14), where $e_{L}$ got one energy power more than $e_{T}$. Due to Eq. (8), the naive leading order $E^{5}$ term of the energy expansion for $\mathcal{T}_{L L T}$ is forced to be zero. As a result, the true leading order is at most $E^{3}$. This is the meaning of the superscript $5 \rightarrow 3$ in Eq. (8). Similar rule applies to other equations. It is important to note that Eqs. (8)-(11) are valid to all loops and are independent of the particles chosen for $v_{1,3,4}$. For the case of string-tree level $\chi=1$ with one tensor $v_{2}$ and three tachyons $v_{1,3,4}$, all four scattering amplitudes in Eq. (11) were calculated to be

$$
\mathcal{T}_{T T T}^{3}=-8 E^{9} \sin ^{3} \phi_{\mathrm{CM}} \mathcal{T}(3)=8 \mathcal{T}_{L L T}^{3}=-8 \mathcal{T}_{(L T)}^{3}=-8 \mathcal{T}_{[L T]}^{3},
$$


where

$$
\begin{aligned}
\mathcal{T}(n)= & \sqrt{\pi}(-1)^{n-1} 2^{-n} E^{-1-2 n}\left(\sin \frac{\phi_{\mathrm{CM}}}{2}\right)^{-3}\left(\cos \frac{\phi_{\mathrm{CM}}}{2}\right)^{5-2 n} \\
& \times \exp \left(-\frac{s \ln s+t \ln t-(s+t) \ln (s+t)}{2}\right),
\end{aligned}
$$

is the high energy limit of

$$
\frac{\Gamma\left(-\frac{s}{2}-1\right) \Gamma\left(-\frac{t}{2}-1\right)}{\Gamma\left(\frac{u}{2}+2\right)}
$$

with $s+t+u=2 n-8$, and we have calculated it up to the next leading order in $E$. This is the order which includes the energy power factor in front of the exponential. Eq. (11) was thus explicitly justified [7,8]. In Eq. (15) $s=-\left(k_{1}+k_{2}\right)^{2}, t=-\left(k_{2}+k_{3}\right)^{2}$ and $u=-\left(k_{1}+\right.$ $\left.k_{3}\right)^{2}$ are the Mandelstam variables and our convention here is different from references [2, $3,11]$ by interchanging $t \leftrightarrow u$.

In deriving Eqs. (8)-(10), we have identified $\mathcal{T}_{\ldots P} \ldots=\mathcal{T}_{\ldots L} \ldots$ not only at the naive leading order but also at the true leading order in energy. For the massless case, this is true since by definitions Eqs. (12) and (13), $e_{P}=e_{L}$. However, for the massive case, it is not obvious that they can be identified. Naively, in the high energy limit, all masses go to zero, and one expects smooth massless limits for all relevant physical quantities and $\mathcal{T}_{\ldots P} \ldots=\mathcal{T}_{\ldots L \ldots}$ as in the massless case. This issue is a more familiar subject in the context of field theories and it turns out that the smooth massless limit may not be achieved for an arbitrary massive field theory. For example, it is well known that the massless limit of a massive gauge field theory is in general different from the massless theory. To illustrate this point, consider a massive gauge field with the Lagrangian density

$$
\mathcal{L}=\frac{1}{4} F_{\mu \nu} F^{\mu \nu}+\frac{m^{2}}{2} A_{\mu} A^{\mu}+A_{\mu} J^{\mu}+\cdots,
$$

where $J$ is a current coupled to $A$ and '...' represents the kinetic term and possible interaction terms for $J$. The equation of motion of $A$ is solved in momentum space as

$$
A_{\mu}=\frac{1}{k^{2}+m^{2}}\left(\eta_{\mu \nu}+\frac{1}{m^{2}} k_{\mu} k_{\nu}\right) J^{v} .
$$

Immediately we see that the massless limit is discontinuous since the second term on the right blows up when $m^{2} \rightarrow 0$. Fronsdal [10] showed that, for vector gauge potentials as well as gauge fields of higher spins, the requirement of the continuity of the massless limit is equivalent to the conservation of charge associated with the gauge symmetry. The second term in Eq. (17) vanishes if we assume charge conservation $k_{\mu} J^{\mu}=0$. Note that the assumption of charge conservation also leads to Ward identities $k_{\mu}\left\langle J^{\mu} \cdots\right\rangle=0$, which are certainly nontrivial relations among correlation functions.

For the cases of our stringy massive states, we do have these stringy gauge symmetries or conserved charges to fulfill Fronsdal's criterion. Therefore, although naively zero-norm states can not by themselves establish nontrivial relations among scattering amplitudes, our assumption about the continuity of the high energy limit, which is implicitly imposed 
when we identify $\mathcal{T}_{\ldots P} \ldots=\mathcal{T}_{\ldots L} \ldots$, leads to nontrivial relations, namely, our stringy Ward identities. In our prescription, zero-norm state is a vehicle used to bring the information about charge conservation to the surface to be seen. As we found explicitly for mass levels $m^{2}=4$ and $m^{2}=6[7,8]$, our prescription indeed leads to ratios between scattering amplitudes for different particles. We give one example here to justify our assumption $\mathcal{T}_{\ldots P} \ldots=\mathcal{T}_{\ldots L \ldots}$. We have explicitly checked that

$$
\mathcal{T}_{P P T}^{3}=\mathcal{T}_{L P T}^{3}=\mathcal{T}_{L L T}^{3}=-E^{9} \sin ^{3} \phi_{\mathrm{CM}} \mathcal{T}(3),
$$

to the leading order in energy, for the case of string tree amplitudes with spin-three tensor $v_{2}$ and three tachyons $v_{1,3,4}$.

\section{Saddle point calculation corrected}

We now briefly review the saddle point calculation of Eq. (3) $[2,3,11]$. First one notes that the high energy limit $\alpha^{\prime} \rightarrow \infty$ is equivalent to the semi-classical limit of first-quantized string theory. In this limit, the closed string $G$-loop scattering amplitudes is dominated by a saddle point in the moduli space $\vec{m}$. For the oriented open string amplitudes, the saddle point configuration can be constructed from an associated configuration of the closed string via reflection principle. It was also found that the Euler number $\chi$ of the oriented open string saddle is always $\chi=1-G$, where $G$ is the genus of the associated closed string saddle. Thus the integral in Eq. (3) is dominated in the $\alpha^{\prime} \rightarrow \infty$ limit by an associated $G$-loop closed string saddle point in $X^{\mu}, \hat{\vec{m}}_{i}$ and $\hat{\xi}_{i}$. The closed string classical trajectory at $G$-loop order was found, according to Gross and Mende [2], to behave at the saddle point as

$$
X_{c 1}^{\mu}(z)=\frac{i}{1+G} \sum_{i=1}^{4} k_{i}^{\mu} \ln \left|z-a_{i}\right|+O\left(\frac{1}{\alpha^{\prime}}\right),
$$

which leads to the $\chi$ th order open string four-tachyon amplitude

$$
\mathcal{T}_{\chi} \approx g^{2-\chi} \exp \left(-\frac{s \ln s+t \ln t+u \ln u}{2(2-\chi)}\right) .
$$

Eq. (20) reproduces the very soft exponential decay $\mathrm{e}^{-\alpha^{\prime} s}$ of the well-known string-tree $\chi=1$ amplitude. There is a consistent check of Eq. (20) at small angles, where the genus$G$ scattering process can be decomposed into $G+1$ successive scatterings [2,12]. The exponent of Eq. (20) can be thought of as the electrostatic energy $E_{G}$ of two-dimensional Minkowski charges $k_{i}$ placed at $a_{i}$ on a Riemann surface of genus $G$. One can use the $S L(2, C)$ invariance of the saddle to fix 3 of the 4 points $a_{i}$, then the only modulus is the cross ratio

$$
\lambda=\frac{\left(a_{1}-a_{3}\right)\left(a_{2}-a_{4}\right)}{\left(a_{1}-a_{2}\right)\left(a_{3}-a_{4}\right)},
$$

which takes the value

$$
\lambda=\hat{\lambda} \approx-\frac{t}{s} \approx \sin ^{2} \frac{\phi_{\mathrm{CM}}}{2}
$$


to extremize $E_{G}$ if we neglect the mass of the tachyons in the high energy limit. For excited string states, it was found that only polarizations in the plane of scattering will contribute to the amplitude at high energies. To the leading order in energy $E$, the products of $e_{T}$ and $e_{L}$ with $\partial^{n} X$ at string-tree level $\chi=1$ (or $G=0$ ) were calculated by using Eq. (19) to be [11]

$$
\begin{aligned}
& e_{T} \cdot \partial^{n} X \sim i(-)^{n} \frac{(n-1) !}{\lambda^{n}} E \sin \phi_{\mathrm{CM}}, \quad n>0, \\
& e_{L} \cdot \partial^{n} X \sim i(-)^{(n-1)} \frac{(n-1) !}{\lambda^{n}} \frac{E^{2} \sin ^{2} \phi_{\mathrm{CM}}}{2 m_{2}} \sum_{l=0}^{n-2} \lambda^{l}, \quad n>1, \\
& e_{L} \cdot \partial^{n} X \sim 0, \quad n=1,
\end{aligned}
$$

where $m_{2}$ is the mass of the particle.

We are now ready to use the results of our zero-norm state calculation, Eqs. (8)-(11), to check the validity of the saddle point calculations, Eqs. (21)-(24). Note that Eqs. (8)(11) are the high energy limit of the decoupling of zero-norm states of stringy scattering amplitudes. They are directly related to the unitarity of string theory. We will just check the string-tree amplitudes since only in this case the exact results are known. Let us use Eqs. (21)-(24) to calculate, for example, the high energy limit of Eq. (4) where both scattering amplitudes $\mathcal{T}^{(\mu \nu \lambda)}, \mathcal{T}^{(\mu \nu)}$ are defined by Eq. (3). It is easy to see, according to Eqs. (21)-(24), that the kinematic factor $\mathcal{K}_{L L T}$ of $\mathcal{T}_{L L T}$ is of energy order $E$ while $\mathcal{K}_{(L T)} \sim E^{3}$. This means that $\mathcal{T}_{L L T}^{3}=0[7,8]$ to the leading order in the saddle point calculation. This obviously violates the result of our zero-norm state calculation Eq. (8), which says $\mathcal{K}_{L L T}^{3}$ and $\mathcal{K}_{(L T)}^{3}$ are of the same energy order $E^{3}$. Since Eq. (8) is the high energy limit of Eq. (4). We conclude that there is an inconsistency between Eq. (4) and Eqs. (21)-(24). In other words, the results (21)-(24) violates the high energy massive gauge invariance [7,8] of Eq. (8) and thus will threat the unitarity of string interactions. Many similar examples with the same inconsistencies exist at higher mass levels mainly due to the wrong result of Eq. (24). They are scattering processes with vertices containing $\partial X^{L}$, or processes whose naive leading order in energy vanishes, e.g., $\mathcal{T}_{L L T}^{5 \rightarrow 3}$.

To further demonstrate why the previous saddle point calculations [2,3,11] fail to be consistent with the stringy Ward identity, we redo the calculations based on the saddle point method and figure out the missing terms in the previous results. Again, we shall use the examples of $m^{2}=4$ amplitudes $\mathcal{T}_{L L T}$ and $\mathcal{T}_{T T T}$ to demonstrate the correct calculation. The spin-three amplitude is defined as

$$
\mathcal{T}^{\mu \nu \lambda} \equiv \int \prod_{i=1}^{4} d x_{i}\left\langle e^{i k_{1} X} \partial X^{\mu} \partial X^{\nu} \partial X^{\lambda} e^{i k_{2} X} e^{i k_{3} X} e^{i k_{4} X}\right\rangle,
$$

and $\mathcal{T}_{L L T}=e_{L}^{\mu} e_{L}^{\nu} e_{T}^{\lambda} \mathcal{T}_{\mu \nu \lambda}$ is calculated to be

$$
\mathcal{T}_{L L T}=\int_{0}^{1} d x x^{-s / 2-1}(1-x)^{-t / 2}\left(e_{L} \cdot k_{1}\right)\left(e_{L} \cdot k_{1}\right)\left(e_{T} \cdot k_{3}\right)
$$




$$
\begin{aligned}
& -2 \int_{0}^{1} d x x^{-s / 2}(1-x)^{-t / 2-1}\left(e_{L} \cdot k_{1}\right)\left(e_{L} \cdot k_{3}\right)\left(e_{T} \cdot k_{3}\right) \\
& +\int_{0}^{1} d x x^{-s / 2+1}(1-x)^{-t / 2-2}\left(e_{L} \cdot k_{3}\right)\left(e_{L} \cdot k_{3}\right)\left(e_{T} \cdot k_{3}\right) .
\end{aligned}
$$

Similarly, for $\mathcal{T}_{T T T}$, we have

$$
\mathcal{T}_{T T T}=\int_{0}^{1} d x x^{-s / 2+1}(1-x)^{-t / 2-2}\left(e_{T} \cdot k_{3}\right)\left(e_{T} \cdot k_{3}\right)\left(e_{T} \cdot k_{3}\right) .
$$

In deriving Eqs. (26)-(29), we have made the $S L(2, R)$ gauge fixing and restricted to the $s-t$ channel amplitude only by choosing $x_{1}=0,0 \leqslant x_{2} \leqslant 1, x_{3}=1, x_{4}=\infty$. Here we need to evaluate three integrals by using saddle point method. For instance, the integral of Eqs. (28) and (29) can be defined as

$$
\begin{aligned}
F_{-1,2} & \equiv \int_{0}^{1} d x x^{-s / 2+1}(1-x)^{-t / 2-2} \\
& =\int_{0}^{1} d x x(1-x)^{-2} \exp \left\{-\frac{s}{2}[\ln x-\tau \ln (1-x)]\right\} \\
& =\int_{0}^{1} d x u_{-1,2}(x) \exp \left\{-\frac{s}{2} f(x)\right\},
\end{aligned}
$$

where we have defined

$$
\begin{aligned}
& \tau \equiv-\frac{t}{s} \simeq \sin ^{2} \frac{\phi_{\mathrm{CM}}}{2}, \\
& u_{a, b}(x) \equiv x^{-a}(1-x)^{-b}, \\
& f(x) \equiv \ln x-\tau \ln (1-x) .
\end{aligned}
$$

The other integrals in Eqs. (26) and (27), $F_{1,0}$ and $F_{0,1}$, can be similarly defined. Note that our definitions of Mandelstam variables here are different from Eqs. (21)-(24) by interchanging $t \leftrightarrow u$. Now, before doing saddle point calculations, we would like to point out that there are two different definitions of the concepts of saddle-points. For the integral of Eq. (30), for example, the first definition is the saddle point $x_{0}$ which leads to $f^{\prime}(x)=0$ and is given by $x_{0}=\frac{1}{1-\tau}$, and we have $f^{\prime \prime}\left(x_{0}\right)=\frac{(1-\tau)^{3}}{\tau}$. In this case, the saddle point is independent of the prefactor $u_{-1,2}$. The more standard definition of saddle point is, however, the value $x_{0}^{\prime}$ which extremizes the exponent of $\exp \left[-\frac{s}{2} f(x)+\ln u_{-1,2}\right]$. In this case, the saddle points of the integrals of the scattering amplitudes of the excited string states will be shifted level by level. We stress that although this shift is of subleading order in energy compared with Eq. (20), its effect will bring down an energy power factor in front 
of the exponential. These power factors are crucial to recover the stringy Ward identities and get the linear relations among high energy scattering amplitudes conjectured by Gross. In the following, we will adopt the first definition to do the calculation. However, both saddle point calculations should give the same results if one does the calculations carefully.

We can use the following formula for a systematic expansion of integral in terms of $\alpha^{\prime}$ :

$$
\begin{aligned}
F\left(\alpha^{\prime}\right) \equiv & \int_{-\infty}^{\infty} d x u(x) \exp \left[-\alpha^{\prime} s f(x)\right] \\
= & u_{0} \exp \left(-\alpha^{\prime} s f_{0}\right) \sqrt{\frac{2 \pi}{\alpha^{\prime} s f_{0}^{\prime \prime}}} \\
& \times\left\{1+\left[\frac{u_{0}^{\prime \prime}}{2 u_{0} f_{0}^{\prime \prime}}-\frac{u_{0}^{\prime} f_{0}^{(3)}}{2 u_{0}\left(f_{0}^{\prime \prime}\right)^{2}}-\frac{f_{0}^{(4)}}{8\left(f_{0}^{\prime \prime}\right)^{2}}+\frac{5\left[f_{0}^{(3)}\right]^{2}}{24\left(f_{0}^{\prime \prime}\right)^{3}}\right] \frac{1}{\alpha^{\prime} s}\right. \\
& \left.+O\left(\frac{1}{\left(\alpha^{\prime} s\right)^{2}}\right)\right\}
\end{aligned}
$$

where $u_{0}, f_{0}, u_{0}^{\prime}, f_{0}^{\prime \prime}$, etc., stand for the values of functions and their derivatives evaluated at $x_{0}$. For simplicity, we only write down the leading and next-to-leading corrections in $1 / \alpha^{\prime}$. One can extend this formula to higher orders such that the desired accuracy can be achieved. Note that the range of integration in Eqs. (26)-(30) can be extended from $(0,1)$ to $(-\infty,+\infty)$ by a change of variable $x \rightarrow \frac{e^{x}}{e^{x}+e^{-x}}$.

Now, in order to avoid complicated expansion of momentum variables $e_{L} \cdot k_{1}$ and $e_{L} \cdot k_{3}$ in the calculations of $\mathcal{T}_{L L T}$ [8], we can use the previous result of $\mathcal{T}_{L L T}=\mathcal{T}_{P P T}$ to the leading order, and calculate

$$
\begin{aligned}
\mathcal{T}_{L L T} & \simeq \mathcal{T}_{P P T} \\
& =\left(\frac{E}{4} \sin \phi_{\mathrm{CM}}\right)\left[F_{1,0}\left(k_{1} \cdot k_{2}\right)^{2}-2 F_{0,1}\left(k_{1} \cdot k_{2}\right)\left(k_{2} \cdot k_{3}\right)+F_{-1,2}\left(k_{2} \cdot k_{3}\right)^{2}\right] \\
& =\left(E^{5} \sin \phi_{\mathrm{CM}}\right)\left[F_{1,0}+2 \tau F_{0,1}+\tau^{2} F_{-1,2}\right], \\
\mathcal{T}_{T T T} & =\left(E \sin \phi_{\mathrm{CM}}\right)^{3} F_{-1,2} .
\end{aligned}
$$

The remaining task for calculating both amplitudes now amounts to the various contributions of the master formula for $F_{1,0}, F_{0,1}$ and $F_{-1,2}$. For $\mathcal{T}_{L L T}$, we notice that $\left.\left(u_{1,0}+2 \tau u_{0,1}+\tau^{2} u_{-1,2}\right)\right|_{x_{0}}=0$, hence both the leading terms and the last two terms in the $1 / \alpha^{\prime}$ corrections of Eq. (35) cancel. After some calculations, we find $\left(u_{1,0}^{\prime}+2 \tau u_{0,1}^{\prime}+\right.$ $\left.\tau^{2} u_{-1,2}^{\prime}\right)\left.\right|_{x_{0}}=0$, so the second term in the $1 / \alpha^{\prime}$ corrections of Eq. (35) sum up to zero. The only surviving contribution comes from the first term in the $1 / \alpha^{\prime}$ corrections in Eq. (35), and we have

$$
\frac{1}{2 f_{0}^{\prime \prime}}\left[u_{1,0}^{\prime \prime}+2 \tau u_{0,1}^{\prime \prime}+\tau^{2} u_{-1,2}^{\prime \prime}\right]_{x_{0}}=\frac{(1-\tau)^{2}}{\tau} .
$$


We emphasize that these are exactly the missing terms of the previous saddle point calculations $[2,3,11]$. Once these corrections are taken into account, ${ }^{1}$ we are able to obtain the result which is consistent with our stringy Ward identities. In fact, we can now use

$$
\sin \phi_{\mathrm{CM}} \simeq 2 \sqrt{\tau(1-\tau)}, \quad \sqrt{\frac{4 \pi}{s f_{0}^{\prime \prime}}}=\frac{1}{E} \sqrt{\frac{\pi \tau}{(1-\tau)^{3}}}
$$

and Eq. (38) to get $\left(\alpha^{\prime} \equiv 1 / 2, s \rightarrow \infty\right)$

$$
\begin{aligned}
\mathcal{T}_{L L T} & =-\left(E^{5} \sin \phi_{\mathrm{CM}}\right)\left(\exp \left\{-\frac{s}{2} f_{0}\right\} \sqrt{\frac{4 \pi}{s f_{0}^{\prime \prime}}}\right) \frac{(1-\tau)^{2}}{\tau} \frac{2}{s} \\
& =-\sqrt{\pi} E^{2} \cos ^{2} \frac{\phi_{\mathrm{CM}}}{2} \exp \left\{-\frac{s}{2} f_{0}\right\} \\
& =-E^{9} \sin ^{3} \phi_{\mathrm{CM}} \mathcal{T}(3) .
\end{aligned}
$$

Similarly, for $\mathcal{T}_{T T T}$, we have only one leading term in Eq. (35), and $\left.u_{-1,2}\right|_{x_{0}}=\frac{1-\tau}{\tau^{2}}$ gives

$$
\begin{aligned}
\mathcal{T}_{T T T} & =-\left(E^{3} \sin ^{3} \phi_{\mathrm{CM}}\right)\left(\exp \left\{-\frac{s}{2} f_{0}\right\} \sqrt{\frac{4 \pi}{s f_{0}^{\prime \prime}}}\right) \frac{(1-\tau)}{\tau^{2}} \\
& =-8 \sqrt{\pi} E^{2} \cos ^{2} \frac{\phi_{\mathrm{CM}}}{2} \exp \left\{-\frac{s}{2} f_{0}\right\} \\
& =-8 E^{9} \sin ^{3} \phi_{\mathrm{CM}} \mathcal{T}(3) .
\end{aligned}
$$

Eqs. (39) and (40) agree with our previous calculations above Eq. (15) based on a different method [7,8]. Finally, by comparing Eq. (39) and Eq. (40), we obtain the desired relation, $\mathcal{T}_{T T T}: \mathcal{T}_{L L T}=8: 1$.

In conclusion, we see that the use of saddle point in Eqs. (19) and (21) is only valid for the tachyons amplitude in Eq. (20). In general, the prediction of Eqs. (21)-(24) gives the right energy exponent in the scattering amplitudes, but not the energy power factors in front of the exponential for the cases of the excited string states. These energy power factors are subleading terms ignored in Eqs. (21)-(24) but they are crucial if one wants to get the linear relations among high energy scattering amplitudes conjectured by Gross [3].

\section{String-tree high energy scattering amplitudes}

In this section, we will first give a general formula of all high energy four-point stringtree amplitudes of arbitrary string states. This formula was first proposed in [7]. Here we will give a general proof and present some examples. Let us begin with the scattering

\footnotetext{
1 Another correction one has to take into account is the saddle point $\hat{\lambda} \approx-\frac{t}{s} \approx \sin ^{2} \frac{\phi_{\mathrm{CM}}}{2}$ in Eq. (21) identified by authors of Refs. [2,3,11]. The saddle point we identified in Eq. (35) is, however, $x_{0}=\frac{1}{1-\tau} \approx \frac{1}{\cos ^{2} \phi_{\mathrm{CM} / 2}}$. Since our convention of Mandelstam variables is different from Refs. [2,3,11] by interchanging $t \leftrightarrow u$, we believe that the correct saddle point of Refs. $[2,3,11]$ should be $\hat{\lambda}_{\text {corr }} \approx \frac{1}{\sin ^{2} \phi_{\mathrm{CM}} / 2}$.
} 


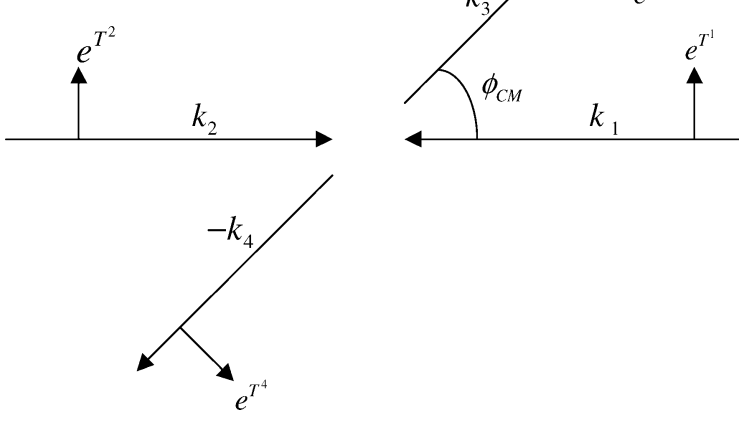

Fig. 1. Kinematic variables in the center of mass frame.

amplitudes of one arbitrary tensor $v_{2}$ and three tachyons $v_{1,3,4}$. Based on the result of high energy stringy Ward identities, it was conjectured in $[7,8]$ that there is only one independent component of high energy scattering amplitude at each fixed mass level, say, $\mathcal{T}_{n}^{T T T} \cdots$ which is defined to be the transverse component of scattering amplitude of the highest spin state at the $m^{2}=2(n-1)$ level, where $n$ is the number of $T$ 's. For example, the first scattering in Eq. (11) $\mathcal{T}_{3}^{T T T}$ corresponds to $m^{2}=4$. All other components of high energy scattering amplitudes are proportional to it. This conjecture was explicitly proved for the mass level $m^{2}=4,6$. It is not difficult to calculate the following general scattering amplitudes of this type with $v_{2}$ the highest spin state at each fixed mass level and three tachyons $v_{1,3,4}$ (we list amplitudes for the $s-t$ channel only)

$$
\mathcal{T}_{n}^{\mu_{1} \mu_{2} \ldots \mu_{n}}=\sum_{l=0}^{n}(-)^{l}\left(\begin{array}{l}
n \\
l
\end{array}\right) B\left(-\frac{s}{2}-1+l,-\frac{t}{2}+n-l\right) k_{1}^{\left(\mu_{1}\right.} \cdots k_{1}^{\mu_{n-l}} k_{3}^{\mu_{n-l+1}} \cdots k_{3}^{\left.\mu_{n}\right)}
$$

where $B(u, v)=\int_{0}^{1} d x x^{u-1}(1-x)^{v-1}$ is the Euler beta function. It is now easy to calculate the general high energy scattering amplitude at the $m^{2}=2(n-1)$ level [8]

$$
\mathcal{T}_{n}^{T T T \cdots}=\left[-2 E^{3} \sin \phi_{\mathrm{CM}}\right]^{n} \mathcal{T}(n),
$$

where $\mathcal{T}(n)$ is given by Eq. (15). One can now generalize this result to multi-tensors [7]

$$
\mathcal{T}_{n_{1} n_{2} n_{3} n_{4}}^{T^{1} \ldots T^{2} \ldots T^{3} \ldots T^{4} \ldots}=\left[-2 E^{3} \sin \phi_{\mathrm{CM}}\right]^{\sum n_{i}} \mathcal{T}\left(n \rightarrow \sum n_{i}\right),
$$

where $n_{i}$ is the number of $T^{i}$ of the $i$ th vertex operators and $T^{i}$ is the transverse direction of the $i$ th particle. This is our first master formula for all scattering amplitudes which are of the leading order in energy compared with other amplitudes at the same mass levels. In proving Eq. (43), one notes that the new contraction terms arising from two $\partial X$ 's belonging to two different vertex operators are suppressed in energy. Finally, the $t-u$ and $s-u$ channels of a given scattering amplitude can be Möbius-transformed to the $s-t$ channel of another scattering amplitude obtained by interchanging vertex operators of the original scattering 
amplitude. Thus we still end up with the $\sin \phi_{\mathrm{CM}}$ behavior in the kinematic factor. This completes our proof for Eq. (43). It is remarkable to find, from Eq. (43), that all high energy scattering amplitudes share this simple high energy behavior, which hints at the universal form of the interactions of an infinite number of string states. In the following we give some examples to illustrate the power of Eq. (43). The scattering amplitudes involving tachyons and massless vectors were calculated in [13], and are given by ( $s-t$ channel only)

$$
\mathcal{T}_{\text {vectors }}=\frac{\Gamma\left(-\frac{s}{2}-1\right) \Gamma\left(-\frac{t}{2}-1\right)}{\Gamma\left(\frac{u}{2}+2\right)} K,
$$

with the kinematic factors

$$
\begin{aligned}
K\left(\zeta_{1},\right. & \left.k_{1} ; \zeta_{2}, k_{2} ; k_{3} ; k_{4}\right) \\
= & \left(\frac{1}{2} t+1\right)\left(\frac{1}{2} u+1\right)\left(\left(\zeta_{1} \cdot k_{2}\right)\left(\zeta_{2} \cdot k_{1}\right)-\zeta_{1} \cdot \zeta_{2}\right) \\
& +\left(\frac{1}{2} s+1\right)\left(\frac{1}{2} u+1\right)\left(\left(\zeta_{1} \cdot k_{4}\right)\left(\zeta_{2} \cdot k_{3}\right)\right) \\
& +\left(\frac{1}{2} s+1\right)\left(\frac{1}{2} t+1\right)\left(\left(\zeta_{1} \cdot k_{3}\right)\left(\zeta_{2} \cdot k_{4}\right)\right) \\
K\left(\zeta_{1},\right. & \left.k_{1} ; \zeta_{2}, k_{2} ; \zeta_{3}, k_{3} ; k_{4}\right) \\
= & \left(\frac{1}{2} s+1\right)\left(\frac{1}{2} t+1\right) \frac{1}{2} t\left(\left(\zeta_{1} \cdot k_{3}\right)\left(\zeta_{2} \cdot k_{1}\right)\left(\zeta_{3} \cdot k_{1}\right)-\left(\zeta_{2} \cdot k_{1}\right)\left(\zeta_{1} \cdot \zeta_{3}\right)\right) \\
& +\left(\frac{1}{2} s+1\right)\left(\frac{1}{2} t+1\right) \frac{1}{2} s\left(\left(\zeta_{2} \cdot k_{3}\right)\left(\zeta_{1} \cdot \zeta_{3}\right)-\left(\zeta_{1} \cdot k_{3}\right)\left(\zeta_{2} \cdot k_{3}\right)\left(\zeta_{3} \cdot k_{1}\right)\right) \\
& +\left(\frac{1}{2} s+1\right)\left(\frac{1}{2} t+1\right)\left(\frac{1}{2} u+1\right)\left(\left(\zeta_{1} \cdot k_{4}\right)\left(\zeta_{2} \cdot k_{1}\right)\left(\zeta_{3} \cdot k_{2}\right)\right. \\
& \left.-\left(\zeta_{1} \cdot k_{2}\right)\left(\zeta_{2} \cdot k_{3}\right)\left(\zeta_{3} \cdot k_{4}\right)-\left(\zeta_{2} \cdot \zeta_{3}\right)\left(\zeta_{1} \cdot k_{2}\right)+\left(\zeta_{1} \cdot \zeta_{2}\right)\left(\zeta_{3} \cdot k_{2}\right)\right) \\
& -\left(\frac{1}{2} t+1\right)\left(\frac{1}{2} u+1\right) \frac{1}{2} t\left(\left(\zeta_{1} \cdot \zeta_{2}\right)\left(\zeta_{3} \cdot k_{4}\right)-\left(\zeta_{1} \cdot k_{2}\right)\left(\zeta_{2} \cdot k_{1}\right)\left(\zeta_{2} \cdot k_{4}\right)\right) \\
& -\left(\frac{1}{2} u+1\right)\left(\frac{1}{2} s+1\right) \frac{1}{2} s\left(\left(\zeta_{1} \cdot k_{4}\right)\left(\zeta_{2} \cdot k_{3}\right)\left(\zeta_{3} \cdot k_{2}\right)-\left(\zeta_{2} \cdot \zeta_{3}\right)\left(\zeta_{1} \cdot k_{4}\right)\right)
\end{aligned}
$$

$K\left(\zeta_{1}, k_{1} ; \zeta_{2}, k_{2} ; \zeta_{3}, k_{3} ; \zeta_{4}, k_{4}\right)$

$$
\begin{aligned}
= & \left(\frac{1}{2} s+1\right)\left(\frac{1}{2} t+1\right)\left(\frac{1}{2} u+1\right) \\
& \times\left[-K^{(s s)}+\frac{1}{2} s\left\{\left(\zeta_{1} \cdot k_{3}\right)\left(\zeta_{2} \cdot k_{3}\right)\left(\left(\zeta_{3} \cdot k_{1}\right)\left(\zeta_{4} \cdot k_{1}\right)+\left(\zeta_{3} \cdot k_{2}\right)\left(\zeta_{4} \cdot k_{2}\right)\right)\right.\right. \\
& \left.+\frac{1}{3}\left(\left(\zeta_{1} \cdot k_{2}\right)\left(\zeta_{2} \cdot k_{3}\right)\left(\zeta_{3} \cdot k_{1}\right)-\left(\zeta_{1} \cdot k_{3}\right)\left(\zeta_{2} \cdot k_{1}\right)\left(\zeta_{3} \cdot k_{2}\right)\right)\left(\zeta_{4} \cdot k_{1}-\zeta_{4} \cdot k_{2}\right)\right\} \\
& +\frac{1}{2} t\left\{\left(\zeta_{2} \cdot k_{1}\right)\left(\zeta_{3} \cdot k_{1}\right)\left(\left(\zeta_{1} \cdot k_{3}\right)\left(\zeta_{4} \cdot k_{3}\right)+\left(\zeta_{1} \cdot k_{2}\right)\left(\zeta_{4} \cdot k_{2}\right)\right)\right.
\end{aligned}
$$




$$
\begin{aligned}
& \left.+\frac{1}{3}\left(\left(\zeta_{1} \cdot k_{3}\right)\left(\zeta_{2} \cdot k_{1}\right)\left(\zeta_{3} \cdot k_{2}\right)-\left(\zeta_{1} \cdot k_{2}\right)\left(\zeta_{2} \cdot k_{3}\right)\left(\zeta_{3} \cdot k_{1}\right)\right)\left(\zeta_{4} \cdot k_{3}-\zeta_{4} \cdot k_{2}\right)\right\} \\
& +\frac{1}{2} u\left\{\left(\zeta_{1} \cdot k_{2}\right)\left(\zeta_{3} \cdot k_{2}\right)\left(\left(\zeta_{2} \cdot k_{1}\right)\left(\zeta_{4} \cdot k_{1}\right)+\left(\zeta_{2} \cdot k_{3}\right)\left(\zeta_{4} \cdot k_{3}\right)\right)\right. \\
& \left.+\frac{1}{3}\left(\left(\zeta_{1} \cdot k_{2}\right)\left(\zeta_{2} \cdot k_{3}\right)\left(\zeta_{3} \cdot k_{1}\right)-\left(\zeta_{1} \cdot k_{3}\right)\left(\zeta_{2} \cdot k_{1}\right)\left(\zeta_{3} \cdot k_{2}\right)\right)\left(\zeta_{4} \cdot k_{3}-\zeta_{4} \cdot k_{1}\right)\right\} \\
& +\frac{s t}{4} \frac{1}{\frac{u}{2}+1}\left(\zeta_{1} \cdot \zeta_{3}-\left(\zeta_{1} \cdot k_{3}\right)\left(\zeta_{3} \cdot k_{1}\right)\right)\left(\zeta_{2} \cdot \zeta_{4}-\left(\zeta_{2} \cdot k_{4}\right)\left(\zeta_{4} \cdot k_{2}\right)\right) \\
& +\frac{s t}{4} \frac{1}{\frac{u}{2}+1}\left(\zeta_{1} \cdot \zeta_{3}-\left(\zeta_{1} \cdot k_{3}\right)\left(\zeta_{3} \cdot k_{1}\right)\right)\left(\zeta_{2} \cdot \zeta_{4}-\left(\zeta_{2} \cdot k_{4}\right)\left(\zeta_{4} \cdot k_{2}\right)\right) \\
& +\frac{s u}{4} \frac{1}{\frac{t}{2}+1}\left(\zeta_{1} \cdot \zeta_{4}-\left(\zeta_{1} \cdot k_{4}\right)\left(\zeta_{4} \cdot k_{1}\right)\right)\left(\zeta_{2} \cdot \zeta_{3}-\left(\zeta_{2} \cdot k_{3}\right)\left(\zeta_{3} \cdot k_{2}\right)\right) \\
& \left.-\frac{s t}{4}\left(\zeta_{1} \cdot \zeta_{3}\right)\left(\zeta_{2} \cdot \zeta_{4}\right)-\frac{t u}{4}\left(\zeta_{1} \cdot \zeta_{2}\right)\left(\zeta_{3} \cdot \zeta_{4}\right)-\frac{s u}{4}\left(\zeta_{1} \cdot \zeta_{4}\right)\left(\zeta_{2} \cdot \zeta_{3}\right)\right]
\end{aligned}
$$

where $K^{(s s)}$ is the same kinematic factor that enters in the type I superstring and can be found in [14]. It is easy to show that $K^{(s s)}$ is suppressed in energy in the high energy expansion. Finally, the 4-point function of a spin-two, a vector and two tachyons is calculated to be

$$
\begin{aligned}
\mathcal{T}_{\text {tensor }}= & \int \prod_{i=1}^{4} d x_{i}\left\langle\zeta_{\mu \nu} \partial X^{\mu} \partial X^{\nu} e^{i k_{1} X} \zeta_{\nu} \partial X^{\nu} e^{i k_{2} X} e^{i k_{3} X} e^{i k_{4} X}\right\rangle \\
= & \frac{\Gamma\left(-\frac{s}{2}-1\right) \Gamma\left(-\frac{t}{2}-1\right)}{\Gamma\left(\frac{u}{2}+2\right)}\left[2\left(\frac{1}{2} t+1\right) \frac{1}{2} u\left(\frac{1}{2} u+1\right)\left(\zeta_{\mu \nu} k_{2}^{\mu} \zeta^{\nu}\right)\right. \\
& -\left(\frac{1}{2} t+1\right) \frac{1}{2} u\left(\frac{1}{2} u+1\right)\left(\zeta_{\mu \nu} k_{2}^{\mu} k_{2}^{\nu}\right)\left(\zeta \cdot k_{1}\right) \\
& +\left(\frac{1}{2} s+1\right) \frac{1}{2} u\left(\frac{1}{2} u+1\right)\left(\zeta_{\mu \nu} k_{2}^{\mu} k_{2}^{\nu}\right)\left(\zeta \cdot k_{3}\right) \\
& -2\left(\frac{1}{2} s+1\right)\left(\frac{1}{2} t+1\right)\left(\frac{1}{2} u+1\right)\left(\zeta_{\mu \nu} k_{3}^{\mu} \zeta^{\nu}\right) \\
& +2\left(\frac{1}{2} s+1\right)\left(\frac{1}{2} t+1\right)\left(\frac{1}{2} u+1\right)\left(\zeta_{\mu \nu} k_{2}^{\mu} k_{3}^{\nu}\right)\left(\zeta \cdot k_{1}\right) \\
& -2\left(\frac{1}{2} s\right)\left(\frac{1}{2} s+1\right)\left(\frac{1}{2} u+1\right)\left(\zeta_{\mu \nu} k_{2}^{\mu} k_{3}^{\nu}\right)\left(\zeta \cdot k_{3}\right) \\
& -\left(\frac{1}{2} s\right)\left(\frac{1}{2} s+1\right)\left(\frac{1}{2} t+1\right)\left(\zeta_{\mu \nu} k_{3}^{\mu} k_{3}^{\nu}\right)\left(\zeta \cdot k_{1}\right) \\
& \left.+\left(\frac{1}{2} s\right)\left(\frac{1}{2} s+1\right)\left(\frac{1}{2} s-1\right)\left(\zeta_{\mu \nu} k_{3}^{\mu} k_{3}^{\nu}\right)\left(\zeta \cdot k_{3}\right)\right] .
\end{aligned}
$$


By using

$$
s=4 E^{2}, \quad t \simeq-4 \sin ^{2} \frac{\phi_{\mathrm{CM}}}{2} E^{2}, \quad u \simeq-4 \cos ^{2} \frac{\phi_{\mathrm{CM}}}{2} E^{2}
$$

in the high energy limit, the transverse components of the high energy limits of Eqs. (45), (46), (47) and (48) are calculated to be

$$
\begin{aligned}
& {\left[-2 E^{3} \sin \phi_{\mathrm{CM}}\right]^{1+1} \mathcal{T}(2), \quad\left[-2 E^{3} \sin \phi_{\mathrm{CM}}\right]^{1+1+1} \mathcal{T}(3),} \\
& {\left[-2 E^{3} \sin \phi_{\mathrm{CM}}\right]^{1+1+1+1} \mathcal{T}(4), \quad\left[-2 E^{3} \sin \phi_{\mathrm{CM}}\right]^{2+1} \mathcal{T}(3) .}
\end{aligned}
$$

These are remarkably consistent with the prediction of Eq. (43).

The second master formula for high energy scattering is valid for processes with vertices not containing $\partial X^{L}$, or equivalently, for those amplitudes whose true leading order in energy are the same as the naive leading order in energy. The high energy string-tree scattering amplitudes of this type with one tensor $v_{2}$ and three tachyons $v_{1,3,4}$ are calculated to be (we list amplitudes for the $s-t$ channel only)

$$
\mathcal{T}_{n}^{\mathrm{sub}}=\prod_{a} \zeta_{a} \cdot\left(\left[-\frac{t}{2}\right]^{l_{a}} k_{1}+\left[\frac{s}{2}\right]^{l_{a}} k_{3}\right) \mathcal{T}(n),
$$

where $\zeta_{a}$, which is either $e^{T}$ or $e^{L}$, corresponds to the polarization of $\partial^{l_{a}} X$ in the vertex operator $v_{2}$ at mass level $m^{2}=2(n-1), n=\sum l_{a}$. For example, $\mathcal{K}_{(L T)}^{3}$, which contains a vertex $\partial X^{(T} \partial^{2} X^{L)}$, can be rewritten as

$$
\begin{aligned}
\mathcal{K}_{(L T)}^{3} & =\left(-\frac{t}{2} k_{1}+\frac{s}{2} k_{3}\right) \cdot e_{(T}\left(\left[-\frac{t}{2}\right]^{2} k_{1}+\left[\frac{s}{2}\right]^{2} k_{3}\right) \cdot e_{L)} \\
& =E^{9} \sin ^{3} \phi_{\mathrm{CM}}
\end{aligned}
$$

which is correctly predicted by Eq. (43). It is straightforward to write down a general formula for the four-tensor scattering amplitudes of these types at arbitrary mass levels. Note that Eq. (49) includes processes that are not the leading high energy scattering amplitudes at each fixed mass level considered in Eq. (43). For example, Eq. (49) gives

$$
\begin{aligned}
\mathcal{K}_{(T T)}^{2} & =\left(-\frac{t}{2} k_{1}+\frac{s}{2} k_{3}\right) \cdot e_{T}\left(\left[-\frac{t}{2}\right]^{2} k_{1}+\left[\frac{s}{2}\right]^{2} k_{3}\right) \cdot e_{T} \\
& =8 E^{8} \sin ^{2} \phi_{\mathrm{CM}},
\end{aligned}
$$

which is not given by Eq. (43). This scattering amplitude is of subleading order in energy at the mass level $m^{2}=4$. Note that the superscripts of $\mathcal{K}_{(L T)}^{3}$ and $\mathcal{K}_{(T T)}^{2}$ represent the naive (or true) leading orders of the scattering amplitudes defined in the paragraph after Eq. (14).

\section{Conclusion}

We have shown that saddle point calculations of high energy string scattering amplitudes of Eqs. (21)-(24) $[2,3,11]$ are not consistent with the zero-norm state calculations of high 
energy stringy Ward identities of Eqs. (8)-(11) [7,8]. In this paper, we have also given the correct saddle point calculation, Eqs. (34)-(40) which are consistent with our previous calculation based on a different method [7,8], to illustrate the importance of subleading energy power factor in front of the exponential of the high energy string amplitude. This power factor is crucial to recover the stringy Ward identities and the linear relations among scattering amplitudes of different string states conjectured by Gross [3].

Based on the tree-level stringy Ward identities derived from the decoupling of two types of zero-norm states, it was conjectured $[7,8]$ that there is only one independent component of high energy scattering amplitude at each fixed mass level. All other components of high energy scattering amplitudes are proportional to it. This conjecture was explicitly proved for the mass levels $m^{2}=4,6[7,8]$. If this conjecture is valid to all higher mass levels, our master formula, Eq. (43), determines all high energy string-tree scattering amplitudes in terms of those of tachyons-another conjecture by Gross [3]. It is worth noting that if all stringy propagating modes contribute at least one high energy scattering amplitude, then Eq. (43) applies to all particles in the string spectrum.

While the importance of zero-norm states in string theory has been largely underestimated, we expect that a clearer understanding of zero-norm states will help us to uncover the fundamental symmetry of string theory.

\section{Acknowledgements}

This work is supported in part by the National Science Council, Taiwan, ROC. We would like to thank Hiroyuki Hata, Hsien-chung Kao, and Yutaka Matsuo for discussions. C.T. and J.C. would also like to thank NCTS/TPE for the hospitality.

\section{References}

[1] D.J. Gross, F. Wilczek, Phys. Rev. Lett. 30 (1973) 1343;

D.J. Gross, F. Wilczek, Phys. Rev. D 8 (1973) 3633;

D.J. Gross, F. Wilczek, Phys. Rev. D 9 (1974) 980;

H.D. Politzer, Phys. Rev. Lett. 30 (1973) 1346.

[2] D.J. Gross, P. Mende, Phys. Lett. B 197 (1987) 129;

D.J. Gross, P. Mende, Nucl. Phys. B 303 (1988) 407.

[3] D.J. Gross, High energy symmetry of string theory, Phys. Rev. Lett. 60 (1988) 1229;

D.J. Gross, High energy symmetry of string theory, Philos. Trans. R. Soc. London A 329 (1989) 401.

[4] J.C. Lee, Phys. Lett. B 241 (1990) 336;

J.C. Lee, Phys. Rev. Lett. 64 (1990) 1636;

J.C. Lee, B. Ovrut, Nucl. Phys. B 336 (1990) 222.

[5] T.D. Chung, J.C. Lee, Phys. Lett. B 350 (1995) 22;

T.D. Chung, J.C. Lee, Z. Phys. C 75 (1997) 555.

[6] J.C. Lee, Prog. Theor. Phys. 91 (1994) 353.

[7] C.T. Chan, J.C. Lee, Stringy symmetries and their high energy limits, hep-th/0312226.

[8] C.T. Chan, J.C. Lee, Nucl. Phys. B 690 (2004) 3, hep-th/0401133.

[9] B. Green, J.H. Schwarz, E. Witten, Superstring Theory, vol. 1, Cambridge Univ. Press, Cambridge, 1988.

[10] C. Fronsdal, Smooth massless limit of field theories, Nucl. Phys. B 167 (1980) 237;

C. Fronsdal, Smooth massless limit of field theories II, Lett. Math. Phys. 4 (1980) 19. 
[11] D.J. Gross, J.L. Manes, The high energy behavior of open string theory, Nucl. Phys. B 326 (1989) 73, see Section 6 for details.

[12] J. Polchinski, String Theory, vol. 1, Cambridge Univ. Press, Cambridge, 1988, Section 9.8.

[13] H. Kawai, D.C. Lewellen, S.-H.H. Tye, Nucl. Phys. B 269 (1986) 1.

[14] J.H. Schwarz, Phys. Rep. 89 (1982) 223. 